\title{
Multichannel Staggered SAR with Reflector Antennas: Discussion and Proof of Concept
}

\author{
Felipe Queiroz de Almeida, Marwan Younis, Gerhard Krieger, Alberto Moreira
}

German Aerospace Center (DLR), Microwaves and Radar Institute

Muenchner Strasse 20, 82234 Wessling, GERMANY

email: felipe.queirozdealmeida@dlr.de

\begin{abstract}
Synthetic Aperture Radar (SAR) spaceborne imaging with High Resolution Wide Swath (HRWS) capabilities has been the subject of increasing interest and active research, driven by applications posing requirements of high spatial resolution and short revisit times which cannot be directly fulfilled with conventional system architectures. In this context, the paper examines a combination of two promising HRWS techniques, namely staggered SAR - a pulse repetition interval variation allowing wide continuous swaths - and a multiple receiver architecture in azimuth - a proven alternative for fine azimuth resolution - with the objective of combining the strengths of both methods. The paper explains the novel multichannel staggered SAR architecture and provides a first proof of concept using data acquired with an experimental ground-based multichannel feed reflector antenna system.
\end{abstract}

\section{Introduction and Motivation}

Synthetic Aperture Radar (SAR) imaging combining high resolution and wide swath (HRWS) has been an active research field [1],[2],[3],[4],[5],[6], owing to the fact that conventional state-of-the-art and near-future spaceborne SAR systems pose challenging requirements for single-channel system architectures [7]. These requirements arise from the multiple scientific applications of SAR data, which in general benefit from high spatial resolution and short revisit times, thus requiring simultaneous imaging of wide swaths over ground [8]. The approach so far has often been the SAR system operation in multiple modes, prioritizing either coverage or resolution (e.g. ScanSAR or spotlight), but multichannel architectures bear the potential to allow simultaneous fulfillment of the requirements and/or added design flexibility.

In this context, the use of multiple channels in combination with digital beamforming (DBF) [1],[2],[3],[4],[5],[6] is considered to be a key technique to enable SAR systems to achieve the needed HRWS capabilities. Several alternatives have been investigated recently. In terms of systems with multiple channels in elevation, a promising architecture includes the use of multiple elevation beams [5],[6] - which allows the imaging of various simultaneous "subswaths" while using beamforming in elevation to distinguish between range-ambiguous returns - in combination with SCan-On-Receive (SCORE) [9] to maximize gain. In a monostatic system, the imaged sub-swaths are however separated by blind ranges, regions where the return signal cannot be recorded since it arrives during the transmission of the pulses, which translates into gaps in the SAR image. This effect can be avoided by the use of staggered $S A R$ [10],[11],[12], in which the pulse repetition interval (PRI) is varied cyclically

The 18th International Radar Symposium IRS 2017, June 28-30, 2017, Prague, 1 Czech Republic 978-3-7369-9343-3 (C2017 DGON 
from pulse to pulse in order to force a controlled migration over range of the blind regions which cannot be recorded. This in turn allows avoiding image gaps by interpolating over azimuth, at the price of an increase of the average pulse repetition frequency (PRF). Performance prediction of systems with this technique has shown very promising results [13], but at present the processing strategy is limited to a single-channel over azimuth, which limits the best achievable azimuth resolution.

Another family of multichannel systems is that with multiple channels in azimuth [2],[3],[5],[6]. Effective processing strategies exist which allow this class of systems to yield very fine azimuth resolution over wide continuous swaths, often at the cost of a large antenna. This characteristic makes this architecture very effective at improving the resolution for a given PRF, but for realistic antenna lengths, the maximum swath width is limited [5],[6].

The goal of the multichannel staggered $S A R$ [14],[15],[16] concept is to combine the strengths of both methods; that is, using SCORE with multiple elevation beams and staggered PRI - beneficial in terms of the swath width and gain - as well as multiple channels in azimuth - which allow improving the resolution. For this, a novel processing strategy is needed, which is briefly described in Section 2 (cf. [15] for a detailed description).

The paper also includes in Section 3 material regarding a proof of concept done with the Microwave and Radar Institute's ground-based MIMO Radar Demonstrator [17],[18],[19]. An actual reflector with a multichannel feed in X-Band embedded on a rail car is employed to acquire radar data over an example scene. The data are acquired in a highly oversampled regular grid, to allow antenna pattern characterization and data calibration. Afterwards, the data are interpolated to simulate a staggered PRI acquisition, using a PRI sequence scaled from a HRWS spaceborne imaging scenario. The data are then reconstructed using the proposed beamforming concept, to allow demonstration of the feasibility of the approach.

Finally, Section 4 reviews and discusses the presented material.

\section{Multichannel Staggered SAR Azimuth Beamforming: Algorithm Description}

The staggered SAR operation [11] means that the PRI is cyclically varied between pulses. This leads the received echoes to be recorded in a periodically non-uniform grid, as indicated in Figure 1. Transmission events still cause certain parts of the receive echo to be lost, what is referred to in the figure as "blockage" and occurs in a range-dependent form [11],[12]. We assume, for a particular range, that $N_{\text {eff }}$ pulses per cycle are received. Additionally, in a multichannel system, a total of $N_{c h}$ independently digitized channels are assumed to be available.

The problem is then how to combine the samples in time domain so that a good approximation of a regular grid with $N_{c h} \cdot N_{\text {eff }}$ samples per cycle can be formed, meaning the samples are regularized while simultaneously increasing the sampling rate, which allows supporting a finer resolution.

The proposed solution for the problem is based on beamforming concepts [20],[21] and involves modelling the multichannel input grid by means of each input sample's antenna pattern

$v_{\text {input }}\left(f_{d}, i\right)=G_{\text {channel }}\left(f_{d}, i \bmod N_{c h}\right) \cdot \exp \left(-j \cdot 2 \cdot \pi \cdot t_{\text {pulse }}\left[i / / N_{c h}\right] \cdot f_{d}\right)$,

for $1 \leq i \leq N_{c h} \cdot N_{e f f}$. In the expression, mod denotes the remainder of integer division, so that $i \bmod N_{c h}$ goes cyclically from 1 to $N_{c h}$ and "//" denotes the result of integer division, 
meaning the index assumes each integer value $N_{c h}$ times before being incremented, as $i$ increases. That is to say, information from the feed channel's antenna pattern $G_{\text {channel }}\left(f_{d}, n\right), 1 \leq n \leq N_{c h}$ is combined with the instants of reception of the pulses $t_{p u l s e}[m], 1 \leq m \leq N_{\text {eff }}$ in Doppler domain $f_{d}$ into an extended array manifold vector with $N_{c h} \cdot N_{\text {eff }}$ elements.

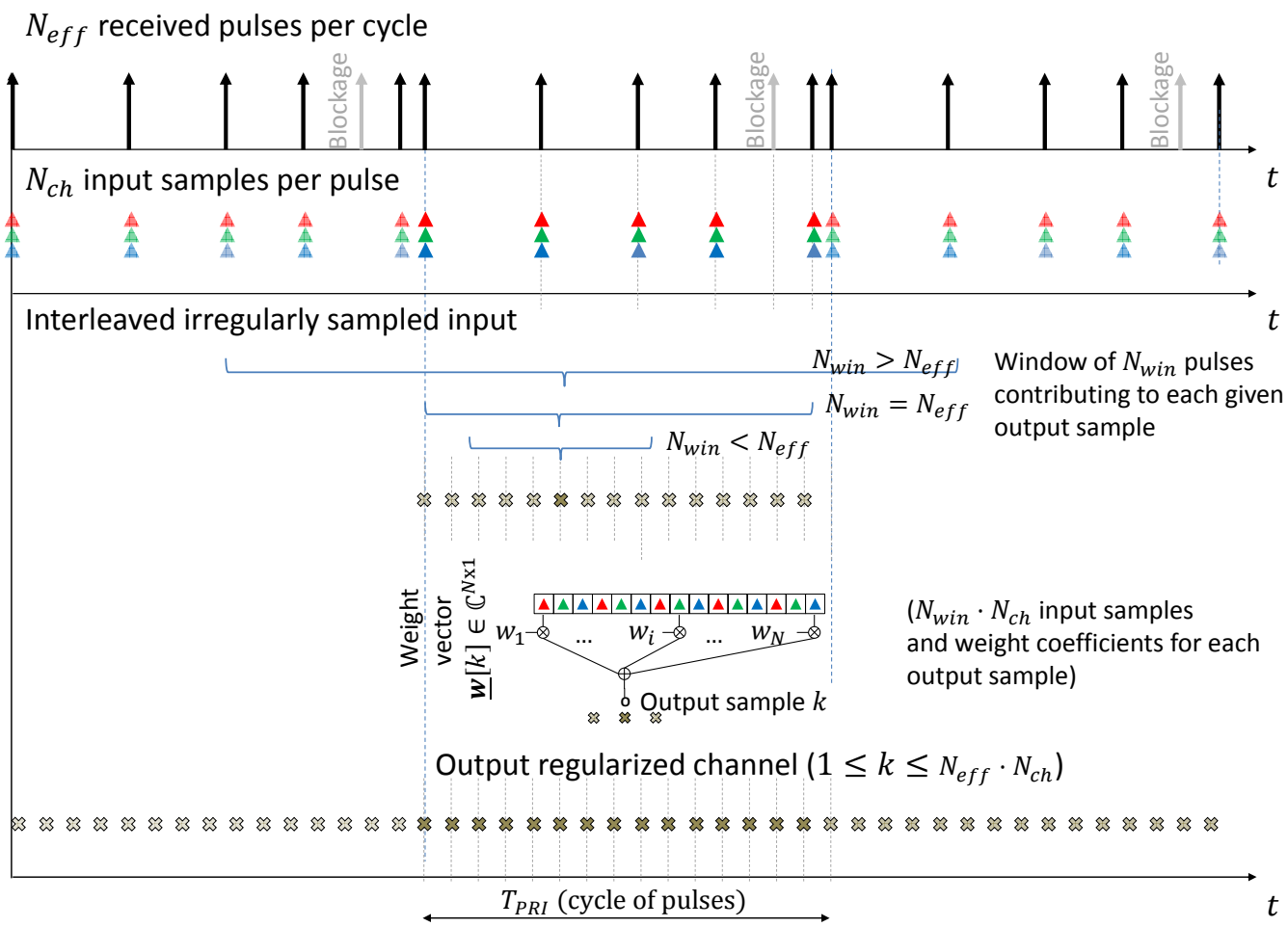

(a)

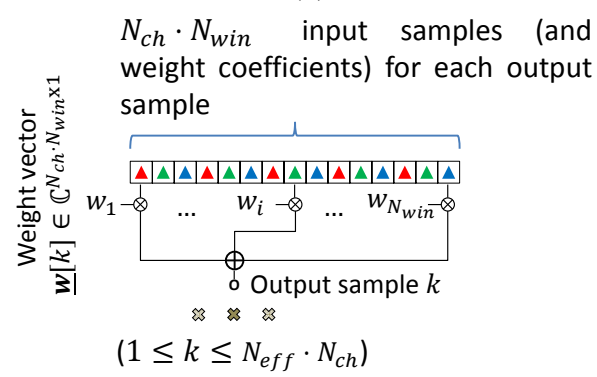

(b)

Figure 1. Required resampling operation for a cycle of pulses with period $T_{P R I}$. (a) provides an overview: the initial periodically non-uniformly sampled grid has $N_{\text {eff }}$ samples per cycle, and the goal is to form a regular grid with $N_{c h} \cdot N_{\text {eff }}$ samples per cycle, achieving simultaneously sample regularization and improvement of the sampling rate by a factor $N_{c h}$, which corresponds to the number of azimuth channels. For this, as highlighted in (b), a total of $N_{c h} \cdot N_{\text {win }}$ samples, including several pulses from the multiple recorded channels are combined by complex weights, derived from beamforming concepts.

The output grid's samples are also modelled by means of each sample's desired antenna pattern, as 
$G_{\text {goal }}\left(f_{d}, k\right)=G_{\text {common }}\left(f_{d}\right) \cdot \exp \left(-j \cdot 2 \cdot \pi \cdot t_{\text {regular }}[k] \cdot f_{d}\right)$,

where $1 \leq k \leq N_{c h} \cdot N_{\text {eff }}$, i.e. a cycle of the output grid. $G_{\text {common }}\left(f_{d}\right)$ is a design parameter (the sum over the feed elements $G_{\text {sum }}\left(f_{d}\right)$ is a meaningful choice for reflector systems [14]) and $t_{\text {regular }}[k]$ should reflect the regularity of the output grid.

The solution for the weights is obtained by minimizing a cost function $\xi\left(G_{\text {goal }}\left(f_{d}, k\right), v_{\text {input }}\left(f_{d}, i\right)\right) ; \quad$ for $1 \leq i \leq N_{c h} \cdot N_{\text {win }}$ and $1 \leq k \leq N_{c h} \cdot N_{\text {eff }}$,

therefore considering both the input and output grids. The cost functions can be flexibly adapted, and different choices of the cost function implement variants of the method. This allows taking into account different criteria, e.g. the degree of regularity of the output grid (closely related to the level of azimuth ambiguity rejection), as well as the final noise scaling of the procedure. As described in [14], [15] in more detail, a meaningful choice for the cost function is

$$
\xi_{J}=(1-\alpha) \cdot \frac{\xi_{M S E}}{n_{M S E}}+\alpha \cdot \frac{n_{S N R}}{\xi_{S N R}},
$$

combining the mean square error (MSE) and signal-to-noise ratio (SNR) cost functions

$$
\begin{aligned}
& \xi_{M S E}=\int\left(G_{\text {goal }}\left(f_{d}, k\right)-\overrightarrow{\mathbf{w}}^{\mathbf{H}} \cdot \overrightarrow{\mathbf{v}_{\text {input }}}\left(f_{d}\right)\right) d f_{d}, \\
& \xi_{S N R}=\frac{\int\left|\overrightarrow{\mathbf{w}}^{\mathbf{H}} \cdot \overrightarrow{\mathbf{v}_{\text {input }}}\left(f_{d}\right)\right|^{2} d f_{d}}{\overrightarrow{\underline{\mathbf{w}}^{\mathbf{H}}} \cdot \overrightarrow{\mathbf{w}}} ;
\end{aligned}
$$

respectively a measure of how closely the implemented patterns approximate a given $G_{\text {goal }}\left(f_{d}, k\right)$ (the solution is obtained for each output sample) and a form of normalized SNR for the pattern. In this formulation, $n_{M S E}$ and $n_{S N R}$ are normalization factors to match the values numerically and adjust sensitivity to the different components, and $\alpha$ is a design parameter in the interval $0 \leq \alpha \leq 1$, to shift emphasis towards pattern fidelity (MSE) or final SNR of the solution. As detailed in [14], [15], (4) can be minimized by applying the complex gradient operator [21] and equating the result to the null vector, yielding a non-linear system of equations that can be solved numerically.

\section{A Proof of Concept with the Radar Demonstrator}

The Microwave and Radar Institute of DLR has developed an experimental multichannel radar system [17],[18] for the demonstration of digital beamforming concepts. The system also allows the usage of reflector antennas [19] and is thus particularly fitted to allow a proof of concept of the multichannel staggered SAR technique, initially intended for this class of system.

To this end, a data acquisition in X-Band was performed over a sandbox with multiple corner reflectors. The data are initially regularly sampled at a high rate of $10.0 \mathrm{~Hz}$, to allow antenna pattern characterization (necessary as input to the weight calculation) and calibration of the data from the 8 independently digitized feed channels. After calibration, the oversampled data are interpolated to achieve a periodically non-uniform grid - as would be the case in a staggered SAR acquisition - with a mean sampling rate of $1.25 \mathrm{~Hz}(1 / 8$ of the original). These data are reconstructed to the original grid (regularly sampled at $10.0 \mathrm{~Hz}$ ) and compared to the data which were regularly sampled in the first place, allowing the assessment of the reconstructed image quality and the feasibility of the approach. 
Figure 2 illustrates the experimental set-up (cf. Figure 2 (a), (b)), showing the corner reflector in the sand box - the scene's main feature; and provides an overview of the processing steps (cf. Figure 2 (c), (d))

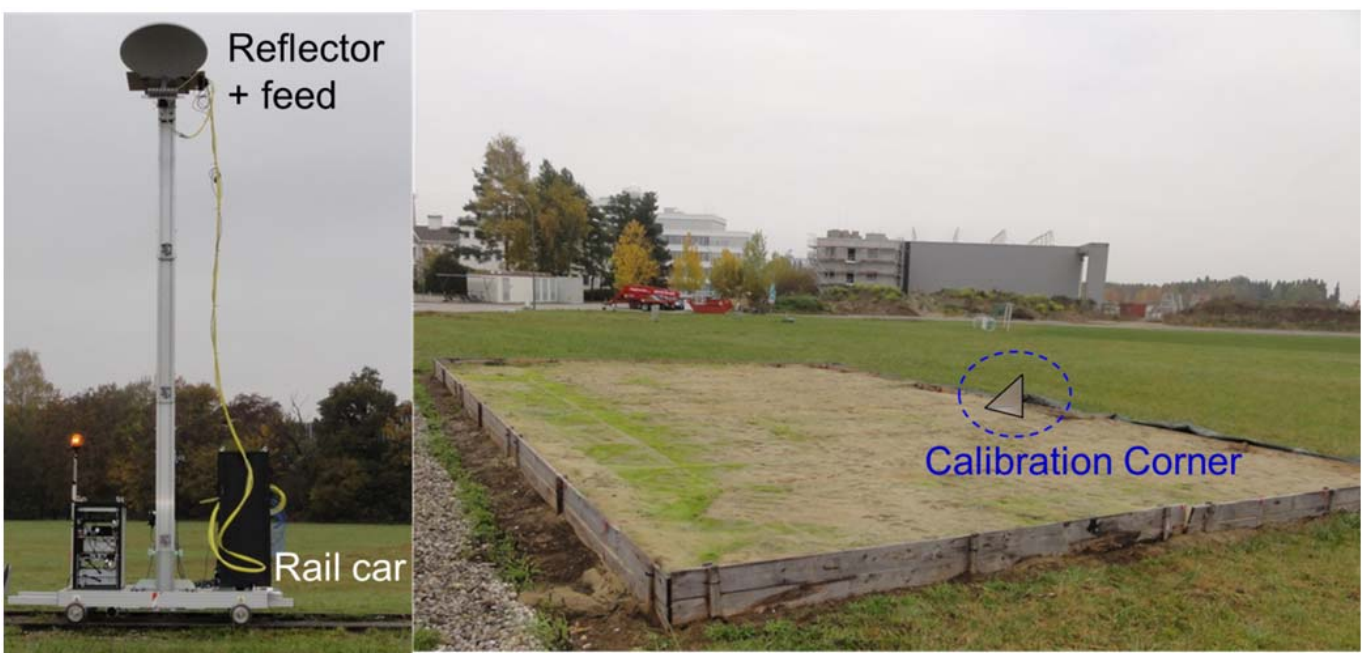

(a)

(b)

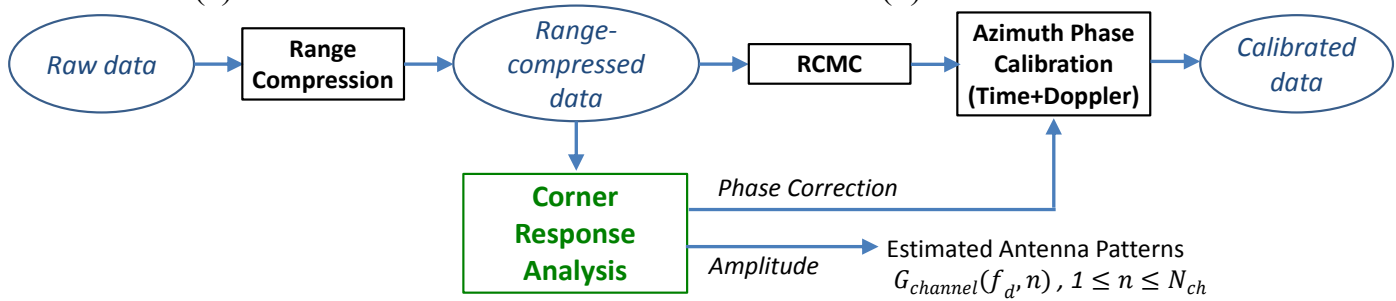

(c)

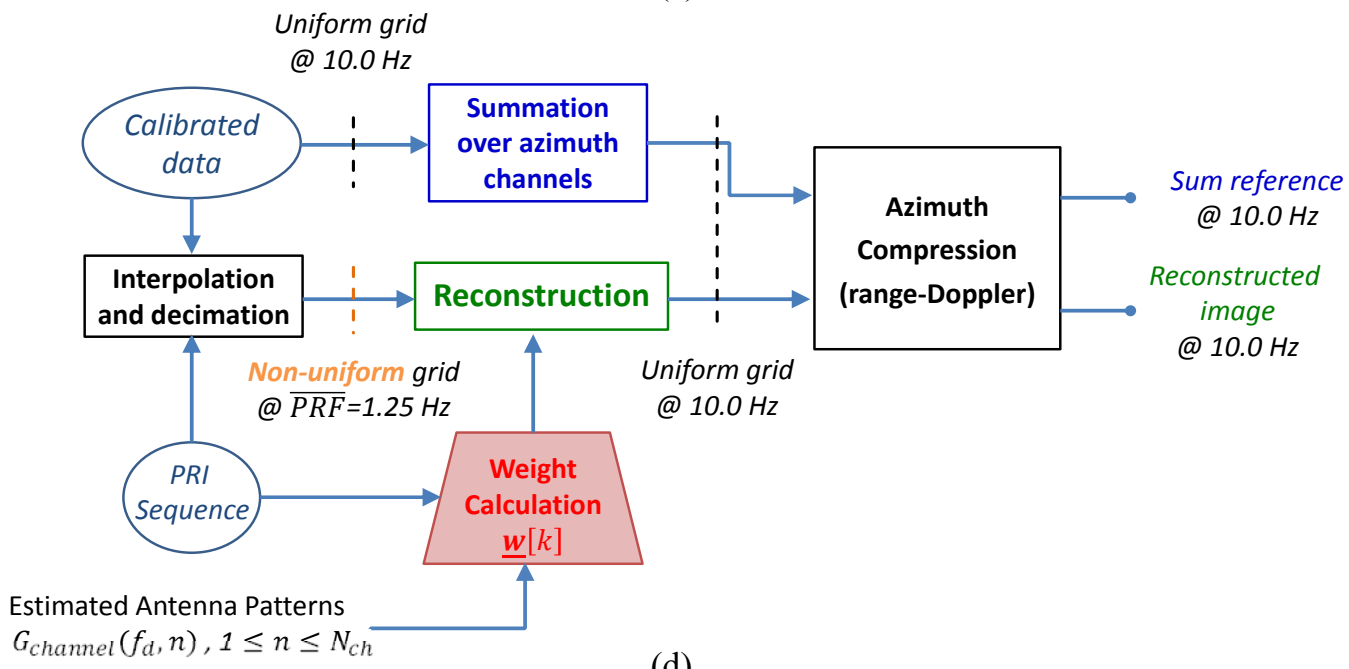

(d)

Figure 2. Experimental set-up and processing for the proof of concept. (a) Radar demonstrator and the reflector antenna. (b) Sandbox and a corner reflector placed as main target. (c) Block diagram for the preprocessing of the data, including analysis of the corner reflector's response, the basis for calibration and estimation of the antenna patterns. (d) Block diagram for the processing involved in the proof of concept, which uses the calibrated data and the antenna pattern information derived before.

Figure 2 (c) provides a basic description of the pre-processing steps needed before the actual proof of concept, described in more detail in Section 3.1. Figure 2 (d) highlights the details of 
the processing chain for the proof of concept itself, detailed in Section 3.2. Finally, Section 3.3 provides the results, comparing the reconstructed image with the reference.

The radar demonstrator system parameters, as well as those related to the proof of concept are summarized in Table 1.

Table 1: Radar demonstrator and proof of concept processing parameters

\begin{tabular}{|c|c|c|}
\hline Quantity & Symbol & Value \\
\hline \multicolumn{3}{|c|}{ Antenna, Pulse and Tx/Rx Hardware Parameters } \\
\hline Center frequency & $f_{0}$ & $9.58 \mathrm{GHz}$ \\
\hline Chirp bandwidth & $B W_{\text {chirp }}$ & $300 \mathrm{MHz}$ \\
\hline Intermediate center frequency & $f_{I F}$ & $205 \mathrm{MHz}$ \\
\hline Peak output power & $P_{T x}$ & $18 \mathrm{dBm}$ \\
\hline ADC sampling rate (real data) & $f_{s}$ & $1 \mathrm{GS} / \mathrm{s}$ \\
\hline ADC resolution & $r_{A D C}$ & 10 bit \\
\hline Elliptical reflector major axis & $D_{m a j}$ & $1.0 \mathrm{~m}$ \\
\hline Elliptical reflector minor axis & $D_{\min }$ & $0.7 \mathrm{~m}$ \\
\hline Reflector focal length & $F_{\text {ref }}$ & $0.5 \mathrm{~m}$ \\
\hline Reflector offset in elevation & $O_{r e f}$ & $0.35 \mathrm{~m}$ \\
\hline Feed element (horn antenna) spacing & $d_{a z}$ & $4.4 \mathrm{~cm}$ \\
\hline Pulse length & $T_{P}$ & $10.0 \mu \mathrm{s}$ \\
\hline System PRF & $P R F$ & $10.0 \mathrm{~Hz}$ \\
\hline Transmitted/Received polarization & - & VV \\
\hline No. of channels in elevation/azimuth & $N_{e l} / N_{a z}$ & $1 / 8$ \\
\hline \multicolumn{3}{|c|}{ Target/Platform Parameters } \\
\hline Antenna height above ground & $h_{e l}$ & $6.34 \mathrm{~m}$ \\
\hline Platform (rail car) velocity & $v_{\text {plat }}$ & $8.5 \mathrm{~cm} / \mathrm{s}$ \\
\hline Calibration corner's ground / slant range & $g_{0} / r_{0}$ & $9.0 \mathrm{~m} / 11.0 \mathrm{~m}$ \\
\hline \multicolumn{3}{|c|}{ Staggered PRI Sequence Parameters (used for interpolation/reconstruction) } \\
\hline Average PRF & $\overline{P R F}$ & $1.25 \mathrm{~Hz}$ \\
\hline Initial PRI & $P R I_{0}$ & $0.891 \mathrm{~s}$ \\
\hline PRI sequence step & $\Delta$ & $-5.31 \mathrm{~ms}$ \\
\hline Sequence length & $N_{P R I}$ & 33 \\
\hline \multicolumn{3}{|c|}{ Grid, Reconstruction and Processing parameters } \\
\hline Output sampling rate & $P R F$ & $10.0 \mathrm{~Hz}$ \\
\hline Number of pulses in azimuth beamformer window & $N_{\text {win }}$ & 7 \\
\hline SNR emphasis parameter & $\alpha$ & 0.1 \\
\hline Maximum phase center shift from input to output grid & $\Delta x_{\max }$ & $3.63 \mathrm{~cm}$ \\
\hline Processed Doppler bandwidth & $B W_{\text {proc }}$ & $3.4 \mathrm{~Hz}$ \\
\hline
\end{tabular}

\subsection{Pre-Processing and Calibration}

Before actually performing the proof of concept, the pre-processing steps listed in Figure 2 (c) are necessary. The radar data are converted to complex I/Q format and range compressed, and these data are the basis for an analysis of the corner reflector's response, as detailed next.

Figure 3 (a) shows the azimuth profile (maxima over range), where the corner reflector is seen in the second half of the scene, and secondary targets in the first. The different channels are plotted in different colors, and the color-code is kept throughout the plots (blue for the first (fore) channel, green for the next and so forth until brown for the aft channel). Figure 3 (b) shows the range history of the corner over the feed channels, against platform position. 


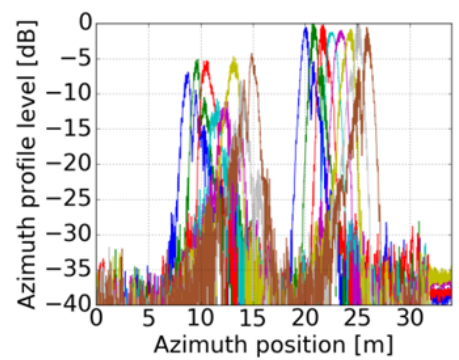

(a)

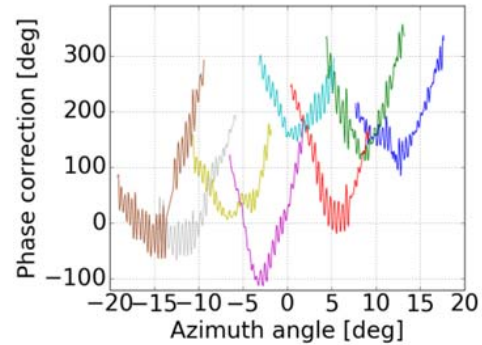

(d)

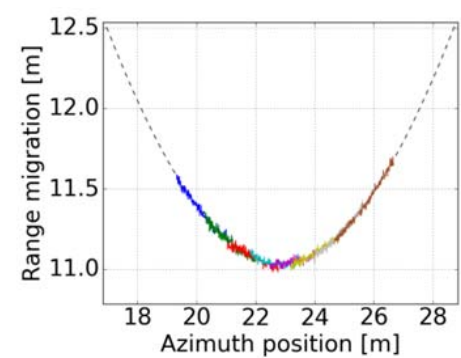

(b)

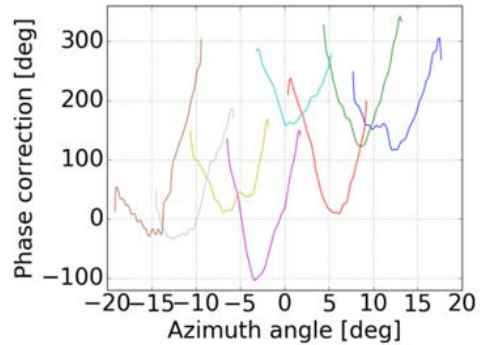

(e)

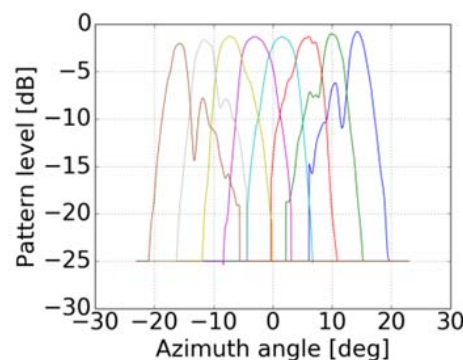

(c)

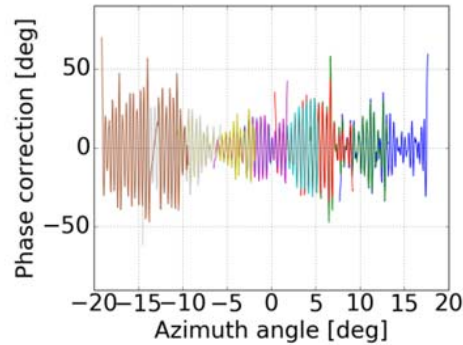

(f)

Figure 3. Corner reflector response analysis and it results. (a) Azimuth profiles of the feed channel's data. (b) Observed range history of corner reflector over different azimuth channels (solid lines) and theoretical values (dashed line). (c) Magnitude of the patterns derived from the corner's response. (d) Azimuth phase correction, discounting the phase expected from the geometry. (e) Low-pass component of the phase correction. (f) Remaining high-pass component of the phase correction.

Figure 3 (c) shows the magnitude of the patterns derived from the data by low-pass filtering the amplitude of the profiles for each channel. Note that the azimuth angle axis is defined to be positive when the platform "looks forward" to the target, which results in the target showing earlier in the image (compare, for example, the fore channel in blue to aft channel in brown). The results correspond to the "Amplitude" information indicated in the diagram of Figure 2 (c) and are later used as inputs to the reconstruction weight calculation. The "Phase correction" (cf. block diagram) is seen in Figure 3 (d), and is derived from the phase of the corner's peak, after removing the phase modulation expected from the theoretical range history (dashed line in Figure 2 (b)). The phase correction is used to calibrate the data after range cell migration correction (RCMC) and is separated into two components. The low pass component (a cut-off frequency of $0.6 \mathrm{~Hz}$ was used) - visible in Figure 3 (e) - is assumed to be caused by the uncalibrated antenna patterns and is corrected in the Doppler domain, relying in the well-known relationship between azimuth angle and Doppler frequency. The remaining high pass component - Figure 3 (f) - is attributed to platform motion and is thus corrected in time domain. The small phase oscillations are in-phase for all feed elements and their frequency (ca. $1 \mathrm{~Hz}$ ) and amplitude (ca. $2 \mathrm{~mm}$ ) match a periodic oscillation of the antenna mast during the data take.

The calibrated data, last step in Figure 2 (c), are the basis for the proof of concept, presented in detail in the next section.

\subsection{Processing Chain for Proof of Concept}

As seen in Figure 2 (d), the calibrated data (cf. Section 3.1) is available in a uniform grid sampled with a PRF of $10 \mathrm{~Hz}$. By summing over the feed elements, data with $G_{\text {sum }}\left(f_{d}\right)$ (cf. Section 2) in this grid are readily available and serve as an upper-quality reference for the reconstruction, referred to as the "sum reference" in the diagram. 
The proof of concept consists in using the calibrated data of each channel to obtain via interpolation a set of non-uniformly sampled data equivalent to a staggered PRI SAR acquisition; and in a next step reconstruct these data to the original regular grid, combining information from all channels according to the algorithm in Section 2.

In the geometry of the ground-based demonstrator, blind ranges are not observable and thus designing a staggered PRI sequence for this geometry is not meaningful. Therefore, a scaled version of a PRI sequence designed for staggered PRI spaceborne imaging is used as an example to generate the non-uniform grid. This sequence (cf. Table 1) has a mean PRF of $1.25 \mathrm{~Hz}$, so that the combination of the 8 channels restores the original $10.0 \mathrm{~Hz}$ sampling. The interpolation step is done by zero padding the data in Doppler domain, with an oversampling factor of 128, and then linearly interpolating between the samples to achieve the desired sampling instants. As a result, the data of each channel are downsampled to a mean PRF of $1.25 \mathrm{~Hz}$.

The weight calculation requires both the PRI sequence parameters and the amplitude of the antenna patterns derived before (cf. Section 3.1) as inputs. As indicated in Table 1, the calculation is done for an SNR emphasis parameter $\alpha=0.1$ and combines the channels over $N_{\text {win }}=7$ pulses at a time to obtain the samples in the output grid. The maximum distance in azimuth between any sample in the input grid and its closest neighbor in the output grid is, in this case, $\Delta x_{\max }=3.63 \mathrm{~cm}$.

Finally, as seen the right hand side of Figure 2 (d), the data are azimuth compressed using the same filters, using the classical range-Doppler algorithm [22]. The results are presented and discussed next in Section 3.3.

\subsection{Results and Discussion}

The results of the reconstruction procedure are summarized in Figure 4. Figure 4 (a) shows the sum reference image, cut around the corner reflector. This corresponds to the upper quality bound, which has exactly the sum pattern $-G_{\text {sum }}\left(f_{d}\right)$ - and is regularly sampled at a rate of $10.0 \mathrm{~Hz}$. Figure 4 (b) shows the same region for the image reconstructed from the nonuniformly sampled data. The regions of high magnitude in the impulse responses are very similar in both images, though small magnitude differences are also visible (e.g. in the region bellow $-30 \mathrm{~dB}$ ) due to the large dynamic range of the plot. This is also seen in Figure 4 (c), where the comparison between the corner's impulse responses in the two images (reconstruction in blue and sum reference in black) shows that the main beams of the responses are almost indistinguishable and the resolution is preserved, whereas some variation of the sidelobe area occurs. This is due to pattern distortions (meaning the average common pattern, discounting the sample's phase center positions in (2), differs from $\left.G_{\text {sum }}\left(f_{d}\right)\right)$ and residual ambiguities (since the achieved output grid is a good approximation of an uniform one, but residual errors in the sampling still exist). Both aspects are analyzed in [14], [15] in more detail.

Figure 4 (d) shows the magnitude of the (complex) difference between the two images. The global maximum of the difference is at a level of $-22 \mathrm{~dB}$ with respect to the maximum of the reference image, whereas the vast majority of the error's magnitude is below $-25 \mathrm{~dB}$. The magnitude of the difference is analyzed in more detail in Figure 4 (e), where the contour lines correspond to the $-20 \mathrm{~dB}$ and $-10 \mathrm{~dB}$ levels of the sum reference image, in order to highlight the position of the main beam of the impulse response. Figure 4 (f) shows the phase of the difference, in which the region where the magnitude of the sum reference is below $-25 \mathrm{~dB}$ is masked out. The same contour lines are repeated, indicating a stable phase with small residual errors in the region of dominant magnitude, which is the most relevant for the signal. 


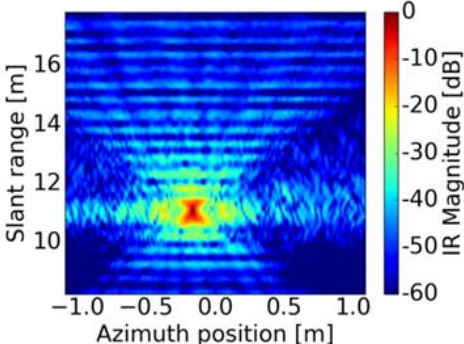

(a)

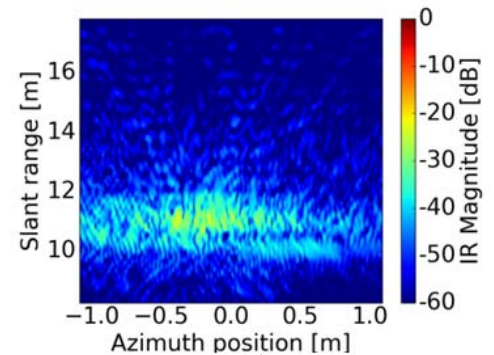

(d)

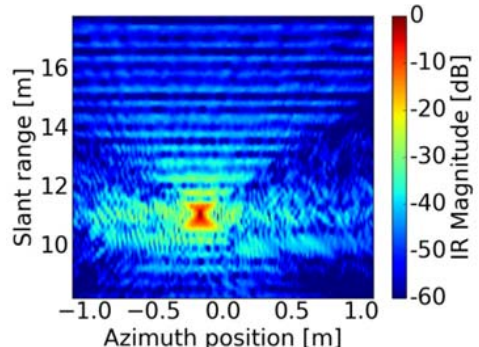

(b)

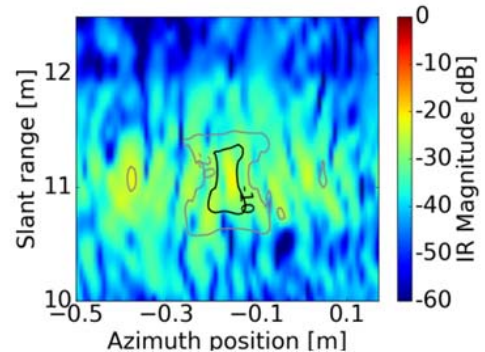

(e)

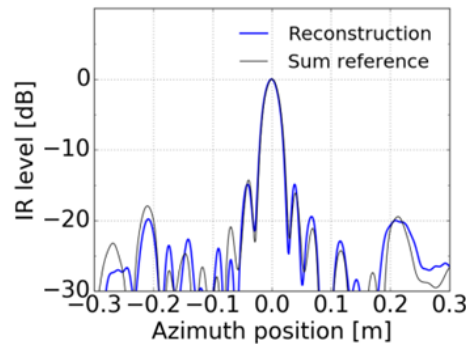

(c)

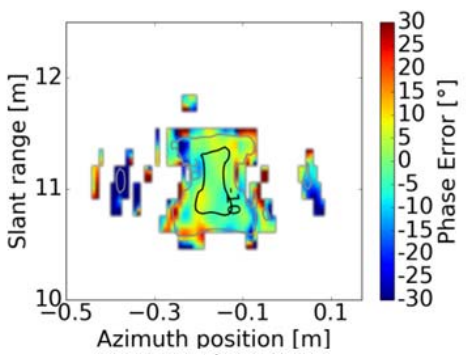

(f)

Figure 4. Results of reconstruction of the data sampled according to the staggered PRI sequence. (a) Sum reference, the upper quality bound. (b) Reconstructed image. (c) Zoom of corner reflector impulse response. (d) Magnitude of the reconstruction error, the (complex) difference between reconstructed image and sum reference. (e) Zoom of the magnitude of reconstruction error, normalized to the maximum of the sum reference. (f) Zoom of the phase of reconstruction error, masked to include only regions where the sum references's amplitude is greater than $-25 \mathrm{~dB}$. The contour lines in (e) and (f) refer to the magnitude levels of the sum reference, as a visual aid to indicate the main region of the impulse response.

In general, the results indicate successful reconstruction using the proposed method, and the achieved error levels are as expected, given the limited accuracy of the radar set-up and calibration procedures.

\section{Final Remarks}

The paper briefly reviewed a new azimuth beamforming algorithm (first introduced in [14] and elaborated on in [15]) suitable for the resampling of the data from reflector antenna systems with multiple channels in azimuth and operated with a staggered-PRI to a uniform grid. The algorithm was applied for a proof of concept on measured data from an experimental ground-based MIMO radar demonstrator system with a reflector in X-band. Azimuth oversampling was used to form a (sparser) non-uniform grid consistent with a staggered PRI sequence and then reconstructed to a uniform grid, allowing a comparison with the original regularly-sampled data. Ancillary calibration and pattern-characterization procedures were also described.

The results indicate successful reconstruction within the bound imposed by calibration accuracy, validating the concept of using the optimal beamforming strategy to influence the sample's phase centers so as to achieve the desired resampling. 


\section{References}

[1] G. Krieger et al., "Advanced Concepts for Ultra-Wide-Swath SAR Imaging," in 7th European Conference on Synthetic Aperture Radar (EUSAR), Friedrichshafen, pp.1-4, 2008.

[2] N. Gebert, G. Krieger and A. Moreira, "Digital Beamforming on Receive: Techniques and Optimization Strategies for High-Resolution Wide-Swath SAR Imaging," IEEE Transactions on Aerospace and Electronic Systems, vol. 45, no. 2, pp.564-592, 2009.

[3] I. Sikaneta, C. H. Gierull and D. Cerutti-Maori, "Optimum Signal Processing for Multichannel SAR: With Application to High-Resolution Wide-Swath Imaging," IEEE Transactions on Geoscience and Remote Sensing, vol. 52, no. 10, pp. 6095-6109, 2014.

[4] S. Huber, A. Patyuchenko, G. Krieger and A. Moreira, "Spaceborne Reflector SAR Systems with Digital Beamforming," IEEE Transactions on Aerospace and Electronic Systems, vol. 48, no. 4, pp.3473-3493, 2012.

[5] G. Krieger et al., "SIMO and MIMO System Architectures and Modes for High-Resolution Ultra-Wide-Swath SAR Imaging," in 11th European Conference on Synthetic Aperture Radar (EUSAR), pp. 1-6., Hamburg, 2016

[6] M. Younis et al., "Techniques and Modes for Multi-Channel SAR Instruments," in 11th European Conference on Synthetic Aperture Radar (EUSAR), pp. 1-6, Hamburg, 2016.

[7] A. Freeman et al., "The "Myth" of the minimum SAR antenna area constraint," IEEE Transactions on Geoscience and Remote Sensing, vol. 38, no. 1, pp. 320-324, Jan 2000.

[8] A. Moreira et al., "Tandem-L: A Highly Innovative Bistatic SAR Mission for Global Observation of Dynamic Processes on the Earth's Surface," IEEE Geoscience and Remote Sensing Magazine, vol. 3, no. 2, pp.8-23, 2015.

[9] M. Suess and W. Wiesbeck, "Side-looking synthetic aperture radar system," European Patent EP 1 241487, Sept., 2002.

[10] Krieger, et al, "Advanced concepts for high-resolution wide-swath SAR imaging," in 8th European Conference on Synthetic Aperture Radar (EUSAR), pp. 1-4, Aachen, 2010.

[11] M. Villano, G. Krieger and A. Moreira: "Staggered SAR: High-Resolution Wide-Swath Imaging by Continuous PRI Variation," IEEE Transactions on Geoscience and Remote Sensing, vol. 52, no.7, pp.4462-4479, 2014.

[12] M. Villano, G. Krieger and A. Moreira, "A Novel Processing Strategy for Staggered SAR," IEEE Geoscience and Remote Sensing Letters, vol. 11, no. 11, pp.1891-1895, 2014

[13] S. Huber et al., "Tandem-L: A Technical Perspective on Future Spaceborne SAR Sensors for Earth Observation" in IEEE Transactions on Geoscience and Remote Sensing, submitted for publication.

[14] F. Queiroz de Almeida and G. Krieger, "Multichannel Staggered SAR Azimuth Sample Regularization," in 11th European Conference on Synthetic Aperture Radar (EUSAR), pp. 1-6, Hamburg, 2016.

[15] F. Queiroz de Almeida, M. Younis, G. Krieger and A. Moreira, "Staggered SAR Azimuth Processing" submitted to IEEE Transactions on Geoscience and Remote Sensing.

[16] F. Queiroz de Almeida, M. Younis, G. Krieger, F. López-Dekker and A. Moreira, "Synthetik-AperturRadarverfahren" German Patent 102016208899, May 2016.

[17] A. Patyuchenko, T. Rommel, P. Laskowski, M. Younis, and G. Krieger, "Digital Beam-Forming Reconfigurable Radar System Demonstrator," in IEEE International Geoscience and Remote Sensing Symposium (IGARSS), pp. 1541-1544, Munich, 2012.

[18] T. Rommel, A. Patyuchenko, P. Laskowski, M. Younis and G. Krieger, "Development of a MIMO Radar System demonstrator - Calibration and demonstration of first results," in 13th International Radar Symposium (IRS), pp. 113-118, Warsaw, 2012.

[19] S. Huber, T. Rommel, A. Patyuchenko and P. Laskowski, "A Reflector Based Digital Beamforming Demonstrator," in 10th European Conference on Synthetic Aperture Radar (EUSAR), pp. 1-4, Berlin, 2014.

[20] C. Balanis, "Arrays: Linear, Planar, and Circular," in Antenna Theory: Analysis and Design, New York: John Wiley \& Sons Inc., 1997.

[21] H. L. V. Trees, Optimum Array Processing, New York: John Wiley \& Sons Inc., 2002.

[22] J. Curlander, R. McDonough, Synthetic Aperture Radar: Systems and Signal Processing, Jon Wiley \& Sons, 1991. 